\title{
GEODÉSICAS NO ESPAÇO HIPERBÓLICO COMPLEXO DE DIMENSÃO 2
}

GEODESIC IN THE COMPLEX HYPERBOLIC SPACE OF DIMENSION 2

JOYCE D. SANTANA ${ }^{\mathrm{a}}$ FRANCISCO DUTENHEFNER ${ }^{\mathrm{b}}$

\begin{abstract}
Resumo
O objetivo principal desse trabalho é definir uma linha geodésica real e uma geodésica complexa no espaço hiperbólico complexo de dimensão 2. Utilizando o modelo da bola explicitamos uma parametrização para os pontos da linha geodésica real que possui dois pontos finais dados na fronteira do espaço hiperbólico complexo. Já para as geodésicas complexas, que são subvariedades totalmente geodésicas de $\mathbb{H}_{\mathbb{C}^{\prime}}^{2}$ além da definição, classificamos a posição relativa de duas delas como ultraparalelas, paralelas e concorrentes.

Palavras-chaves: espaço hiperbólico complexo, linha geodésica real, geodésica complexa
\end{abstract}

\begin{abstract}
The main objective of this work is to define a real geodesic line and a complex geodesic in complex hyperbolic space of dimension 2 . Using the ball model we explain a parametrization to the points of the actual geodesic line that has two endpoints given at the boundary of complex hyperbolic space. For complex geodesics, which are totally geodesic submanifold of $\mathbb{H}_{\mathbb{C}^{\prime}}^{2}$ in addition to the definition, we classify the relative position of two of them as ultra-parallel, parallel and concurrent.
\end{abstract}

Key-words: complex hyperbolic space, real geodesic line, complex geodesic

MSC2010: 51M10

${ }^{\text {a}}$ Universidade Federal de Minas Gerais - UFMG, Belo Horizonte, MG, Brasil; ORCID: https://orcid.org/ 0000-0003-4867-3012; E-mail: joyce.jds@gmail.com

${ }^{b}$ Universidade Federal de Minas Gerais - UFMG, Belo Horizonte, MG, Brasil; E-mail: chico@mat.ufmg.br 


\section{Introdução}

Segundo Parker [3], abordar o espaço hiperbólico complexo de dimensão 2 é vantajoso já que a maioria dos resultados se generalizam naturalmente para qualquer dimensão. Indo além, Goldman [1] fez um estudo aprofundado do espaço hiperbólico complexo de dimensão $n$, apontando as generalizações entre o espaço hiperbólico complexo de dimensão 2 e dimensão $n$. Para o presente trabalho, que visa definir dois objetos no espaço hiperbólico complexo de dimensão 2 , os resultados principais serão apresentados apenas para dimensão 2. Maiores detalhes sobre o assunto podem ser encontrados em [5].

Definiremos três modelos de $\mathbb{H}_{\mathbb{C}^{\prime}}^{2}$ o modelo projetivo, o modelo da bola unitária e o modelo do parabolóide (ou domínio de Siegel).

\subsection{Formas hermitianas em $\mathbb{C}^{2,1}$}

Considere $H=\left(a_{i j}\right)$ uma matriz complexa $k \times l$. Definimos a transposta hermitiana de $H$ como sendo a matriz complexa $H^{*}=\left(\bar{a}_{j i}\right)$ cujas entradas são os conjugados das entradas da matriz $H$ transposta.

A matriz complexa $H, k \times k$, é dita hermitiana se $H=H^{*}$.

Para cada matriz $k \times k$ hermitiana $H$ podemos associar uma forma hermitiana

$$
\langle\cdot, \cdot\rangle: \mathbb{C}^{k} \times \mathbb{C}^{k} \rightarrow \mathbb{C}
$$

dada por $\langle Z, W\rangle=W^{*} H Z$ em que $W$ e $Z$ são vetores coluna em $\mathbb{C}^{k}$.

As formas hermitianas são lineares em relação a primeira coordenada e linear conjugada em relação a segunda coordenada.

Propriedades: Para $Z, Z_{1}, Z_{2}, W$ vetores coluna de $\mathbb{C}^{k}$ temos:

1. $\left\langle Z_{1}+Z_{2}, W\right\rangle=\left\langle Z_{1}, W\right\rangle+\left\langle Z_{2}, W\right\rangle$

2. $\langle\lambda Z, W\rangle=\lambda\langle Z, W\rangle$

3. $\langle Z, W\rangle=\overline{\langle W, Z\rangle}$

Uma prova dessas propriedades pode ser encontrada em ([3], p. 3). 
Das propriedades acima podemos verificar que

$$
\begin{aligned}
\langle Z, Z\rangle & \in \mathbb{R} \\
\langle Z, \lambda W\rangle & =\bar{\lambda}\langle Z, W\rangle \\
\langle\lambda Z, \lambda W\rangle & =|\lambda|^{2}\langle Z, W\rangle \\
|\langle Z, W\rangle|^{2} & =\langle Z, W\rangle\langle W, Z\rangle
\end{aligned}
$$

De fato, como $\langle Z, W\rangle=\overline{\langle W, Z\rangle}$, então $\langle Z, Z\rangle=\overline{\langle Z, Z\rangle}$ e $\langle Z, Z\rangle \in \mathbb{R}$. Pela definição, $\langle Z, \lambda W\rangle=(\lambda W)^{*} H Z=\bar{\lambda} W^{*} H Z=\bar{\lambda}\langle Z, W\rangle$. Por este último resultado e sabendo que $\langle\lambda Z, W\rangle=\lambda\langle Z, W\rangle$, temos que $\langle\lambda Z, \lambda W\rangle=\lambda \bar{\lambda}\langle Z, W\rangle=|\lambda|^{2}\langle Z, W\rangle$. Por fim, como para qualquer número complexo $z$ vale $z \bar{z}=|z|^{2}$ e $\langle Z, W\rangle=\overline{\langle W, Z\rangle}$, então $\langle Z, W\rangle\langle W, Z\rangle=|\langle Z, W\rangle|^{2}$.

Seja $\mathbb{C}^{2,1}$ o espaço vetorial complexo $\mathbb{C}^{3}$ munido com a forma hermitiana não degenerada (núcleo trivial) e indefinida (admite valores positivos, negativos e eventualmente nulos) de assinatura $(2,1)$. Assim, $\langle\cdot, \cdot\rangle$ é dada por uma matriz hermitiana com 2 autovalores positivos e 1 autovalor negativo. Veja detalhes em ([8], p. 18), e ([3], p. 3).

Existem algumas formas hermitianas que são bastante usadas. Como exemplo, destacaremos três.

$$
\begin{gathered}
\text { Sejam } Z=\left[\begin{array}{l}
z_{1} \\
z_{2} \\
z_{3}
\end{array}\right] \text { e } W=\left[\begin{array}{l}
w_{1} \\
w_{2} \\
w_{3}
\end{array}\right] \in \mathbb{C}^{2,1} \text {. A forma hermitiana usual é definida por } \\
\langle Z, W\rangle=z_{1} \overline{w_{1}}+z_{2} \overline{w_{2}}-z_{3} \overline{w_{3}}
\end{gathered}
$$

cuja matriz hermitiana é:

$$
H_{1}=\left(\begin{array}{ccc}
1 & 0 & 0 \\
0 & 1 & 0 \\
0 & 0 & -1
\end{array}\right)
$$

A forma hermitiana definida por

$$
\langle Z, W\rangle=z_{1} \overline{w_{3}}+z_{2} \overline{w_{2}}+z_{3} \overline{w_{1}}
$$

tem matriz hermitiana:

$$
H_{2}=\left(\begin{array}{lll}
0 & 0 & 1 \\
0 & 1 & 0 \\
1 & 0 & 0
\end{array}\right)
$$


E a forma hermitiana dada por

$$
\langle Z, W\rangle=z_{1} \overline{w_{1}}-z_{2} \overline{w_{3}}-z_{3} \overline{w_{2}}
$$

tem a seguinte matriz hermitiana:

$$
H_{3}=\left(\begin{array}{ccc}
1 & 0 & 0 \\
0 & 0 & -1 \\
0 & -1 & 0
\end{array}\right)
$$

Como explica Parker ([3], p. 4), podemos usar qualquer forma hermitiana de assinatura $(2,1)$, já que $\mathbb{C}^{2,1}$ depende apenas da assinatura e não da forma hermitiana, sendo outras formas, não apresentadas aqui, amplamente utilizadas na literatura.

\subsection{Modelos do espaço hiperbólico complexo de dimensão 2}

Podemos definir os seguintes subconjuntos de $\mathbb{C}^{2,1}$ :

$$
\begin{aligned}
& V_{+}=\left\{Z \in \mathbb{C}^{2,1} \mid\langle Z, Z\rangle>0\right\} \\
& V_{0}=\left\{Z \in \mathbb{C}^{2,1} \mid\langle Z, Z\rangle=0\right\} \\
& V_{-}=\left\{Z \in \mathbb{C}^{2,1} \mid\langle Z, Z\rangle<0\right\}
\end{aligned}
$$

Os vetores pertencentes a $V_{+}$são chamados de vetores positivos, os vetores em $V_{0}$ são nomeados vetores nulos ou isotrópicos e os vetores de $V_{\text {- são ditos }}$ vetores negativos.

\subsubsection{Modelo Projetivo}

Defina em $\mathbb{C}^{2,1}-\{0\}$ a relação de equivalência

$$
Z \sim W \Leftrightarrow W=\lambda Z
$$

onde $\lambda$ é um escalar complexo não nulo.

Seja $\mathbb{C} \mathbb{P}^{2}$ o conjunto das classes dessa relação de equivalência e seja $\mathbb{P}: \mathbb{C}^{2,1}$ $\{0\} \rightarrow \mathbb{C P}^{2}$ a projeção natural dada por $\mathbb{P}(Z)=[Z]$, em que [Z] é a classe de equivalência de $Z$.

O modelo projetivo do espaço hiperbólico complexo é visto como o conjunto das linhas negativas em $\mathbb{C}^{2,1}$, ou seja, $\mathbb{H}_{\mathbb{C}}^{2}=\mathbb{P}\left(V_{-}\right)$e sua fronteira como a conjunto 
das linhas nulas, isto é, $\partial \mathbb{H}_{\mathbb{C}}^{2}=\mathbb{P}\left(V_{0}\right)$. Ao conjunto das linhas positivas denotaremos por $\ell \mathbb{H}_{\mathbb{C}}^{2}=\mathbb{P}\left(V_{+}\right)$.

No modelo projetivo, Goldman ([1], p. 76) define a métrica em $\mathbb{H}_{\mathbb{C}^{\prime}}^{2}$ chamada de métrica de Bergman, sendo esta dada pela função distância $\rho(\cdot, \cdot)$ por

$$
\cosh ^{2}\left(\frac{\rho(z, w)}{2}\right)=\frac{\langle Z, W\rangle\langle W, Z\rangle}{\langle Z, Z\rangle\langle W, W\rangle}
$$

onde $z, w \in \mathbb{H}_{\mathbb{C}}^{2}$ e $Z, W$ são representantes de $z$ e $w$ em $\mathbb{C}^{2,1}$, respectivamente. Observe que esta fórmula independe da escolha dos representantes de $z$ e $w$ em $\mathbb{C}^{2,1}$.

\subsubsection{Modelo da bola}

Considere $Z \in V_{-} \subset \mathbb{C}^{2,1}$ e a forma hermitiana $\langle Z, W\rangle=z_{1} \overline{w_{1}}+z_{2} \overline{w_{2}}-z_{3} \overline{w_{3}}$. Então, $\langle Z, Z\rangle=z_{1} \overline{z_{1}}+z_{2} \overline{z_{2}}-z_{3} \overline{z_{3}}=\left|z_{1}\right|^{2}+\left|z_{2}\right|^{2}-\left|z_{3}\right|^{2}<0$. Assim, $z_{3} \neq 0$. Logo, dividindo todas as coordenadas de $Z$ por $z_{3}$ obtemos um vetor negativo na mesma linha negativa determinada por $Z$ :

$$
\left[\begin{array}{c}
z_{1} / z_{3} \\
z_{2} / z_{3} \\
1
\end{array}\right]
$$

Nomeando $z_{1}^{\prime}=z_{1} / z_{3}$ e $z_{2}^{\prime}=z_{2} / z_{3}$, vemos que o vetor $\left[\begin{array}{l}z_{1}^{\prime} \\ z_{2}^{\prime}\end{array}\right]$ é tal que

$$
\left|z_{1}^{\prime}\right|^{2}+\left|z_{2}^{\prime}\right|^{2}=\left|\frac{z_{1}}{z_{3}}\right|^{2}+\left|\frac{z_{2}}{z_{3}}\right|^{2}=\frac{\left|z_{1}\right|^{2}+\left|z_{2}\right|^{2}}{\left|z_{3}\right|^{2}}<1
$$

Logo, $\left[\begin{array}{l}z_{1}^{\prime} \\ z_{2}^{\prime}\end{array}\right]$ pertence a bola unitária $\mathbb{B}^{2}=\left\{\left.\left[\begin{array}{l}x \\ y\end{array}\right] \in \mathbb{C}^{2}|| x\right|^{2}+|y|^{2}<1\right\}$.

Por outro lado, dado um ponto $\left[\begin{array}{l}x \\ y\end{array}\right] \in \mathbb{B}^{2}$, pode-se definir o vetor negativo $\left[\begin{array}{l}x \\ y \\ 1\end{array}\right] \in \mathbb{C}^{2,1}$.

Assim, o espaço hiperbólico complexo pode ser identificado com a bola $\mathbb{B}^{2}$. A 
fronteira do modelo da bola unitária é dada por

$$
\partial \mathbb{B}^{2}=\left\{\left.\left[\begin{array}{l}
x \\
y
\end{array}\right] \in \mathbb{C}^{2}|| x\right|^{2}+|y|^{2}=1\right\}
$$

que é identificada com a esfera $\mathbb{S}^{3}$.

Dado um ponto $z=\left(z_{1}, z_{2}\right) \in \mathbb{B}^{2}$, o levantamento ou representante de $z$ é o ponto $Z \in \mathbb{C}^{2,1}$ dado por

$$
Z=\left[\begin{array}{c}
z_{1} \\
z_{2} \\
1
\end{array}\right]
$$

De modo geral, sempre utilizamos esse modelo de levantamento, mas, em ocasiões específicas, pode ser necessário a utilização de outros levantamentos.

\subsubsection{Domínio de Siegel ou modelo do parabolóide}

Para $Z, W \in \mathbb{C}^{2,1}$, considere a seguinte forma hermitiana:

$$
\langle Z, W\rangle=z_{1} \overline{w_{1}}-z_{2} \overline{w_{3}}-z_{3} \overline{w_{2}}
$$

Se $Z \in V_{-}$então:

$$
\begin{aligned}
\langle Z, Z\rangle & =z_{1} \overline{z_{1}}-z_{2} \overline{z_{3}}-z_{3} \overline{z_{2}}<0 \\
& \Rightarrow 2 \operatorname{Re}\left(z_{2} \overline{z_{3}}\right)-\left|z_{1}\right|^{2}>0
\end{aligned}
$$

Assim o conjunto dos pontos que satisfaz (1.1) pode ser identificado com o espaço hiperbólico complexo.

Fazendo $z_{3}=1$ obtemos o seguinte conjunto

$$
\mathfrak{h}^{2}=\left\{\left.\left[\begin{array}{c}
z_{1} \\
z_{2}
\end{array}\right] \in \mathbb{C}^{2}\left|2 \operatorname{Re}\left(z_{2}\right)-\right| z_{1}\right|^{2}>0\right\}
$$

que é chamado domínio de Siegel.

A fronteira do domínio de Siegel é dada por

$$
\partial \mathfrak{h}^{2}=\left\{\left.\left[\begin{array}{c}
z_{1} \\
z_{2}
\end{array}\right] \in \mathbb{C}^{2}\left|2 \operatorname{Re}\left(z_{2}\right)-\right| z_{1}\right|^{2}=0\right\} \cup\left\{p_{\infty}\right\}
$$


em que $p_{\infty}$ é o ponto ideal, cujo levantamento em $\mathbb{C}^{2,1}$ é dado por $P_{\infty}=\left[\begin{array}{l}0 \\ 1 \\ 0\end{array}\right]$.

O ponto $o=\left[\begin{array}{l}0 \\ 0\end{array}\right] \in \partial \mathfrak{h}^{2}$ é a origem do domínio de Siegel. Um levantamento em $\mathbb{C}^{2,1}$ para este ponto pode ser o vetor $O=\left[\begin{array}{l}0 \\ 0 \\ 1\end{array}\right]$.

Como $2 \operatorname{Re}\left(z_{2}\right)-\left|z_{1}\right|^{2}=0$ representa um parabolóide, o modelo do domínio de Siegel também é conhecido como modelo do parabolóide.

Parker ([3], p. 8) explicita uma isometria, chamada de Transformação de Cayley, de pontos no modelo da bola em pontos no modelo do parabolóide. Isso nos garante que os resultados obtidos usando um dos modelos será mantido no outro.

\subsection{O conjunto $U(2,1)$}

Seja $U(2,1)$ o conjunto das matrizes complexas $3 \times 3$ que preservam uma forma hermitiana dada em $\mathbb{C}^{2,1}$. Isto é, $A \in U(2,1)$ se

$$
\langle A Z, A W\rangle=\langle Z, W\rangle,
$$

para quaisquer vetores coluna $Z, W \in \mathbb{C}^{2,1}$.

Cada matriz em $U(2,1)$ que é um múltiplo escalar complexo não nulo da identidade, leva cada linha de $\mathbb{C}^{2,1}$ nela mesma, assim age trivialmente no espaço hiperbólico complexo. Considere a aplicação $\mathbb{P}: U(2,1) \rightarrow A u t\left(\mathbb{C P}^{2}\right)$, onde $A u t\left(\mathbb{C P}^{2}\right)$ é o grupo dos biholomorfismos de $\mathbb{C P}^{2}$. Definimos o grupo unitário projetivo $P U(2,1)=\mathbb{P}(U(2,1))$ sendo o grupo dos biholomorfismos de $\mathbb{H}_{\mathbb{C}^{\prime}}^{2}$ cujos elementos são chamados de transformações lineares projetivas.

Se $A \in U(2,1)$ então $A$ é uma matriz complexa $3 \times 3$ tal que $\langle A Z, A W\rangle=\langle Z, W\rangle$ para quaisquer $Z, W \in \mathbb{C}^{2,1}$. Se $H$ é a matriz da forma hermitiana dada em $\mathbb{C}^{2,1}$ então esta equação implica que

$$
(A W)^{*} H(A Z)=W^{*} H Z \Rightarrow W^{*}\left(A^{*} H A\right) Z=W^{*} H Z \text {. }
$$

Como $W$ e $Z$ são arbitrários, para a última igualdade ser verdadeira devemos ter

$$
A^{*} H A=H .
$$


Portanto, $U(2,1)$ também pode ser caracterizado por:

$$
U(2,1)=\left\{A \in M_{3 \times 3}(\mathbb{C}) \mid A^{*} H A=H\right\}
$$

Multiplicando a expressão (1.2) por $H^{-1}$ obtemos $\left(H^{-1} A^{*} H\right) A=I d$, e, temos a seguinte expressão conveniente para a inversa de uma matriz $A \in U(2,1)$ :

$$
A^{-1}=H^{-1} A^{*} H
$$

A propriedade abaixo facilita o cálculo do produto $A^{*} H A$ quando se deseja verificar que uma dada matriz $A$ é hermitiana.

Propriedade 1.1. Seja $H$ uma matriz hermitiana $3 \times 3$ e seja $\langle Z, W\rangle=W^{*} H Z$ a forma hermitiana definida por $H$ em $\mathbb{C}^{3}$. Se $A$ é uma matriz $3 \times 3$ cujas colunas são, respectivamente, os vetores $V_{1}, V_{2}$ e $V_{3}$ então

$$
A^{*} H A=\left[\begin{array}{ccc}
\left\langle V_{1}, V_{1}\right\rangle & \left\langle V_{2}, V_{1}\right\rangle & \left\langle V_{3}, V_{1}\right\rangle \\
\left\langle V_{1}, V_{2}\right\rangle & \left\langle V_{2}, V_{2}\right\rangle & \left\langle V_{3}, V_{2}\right\rangle \\
\left\langle V_{1}, V_{3}\right\rangle & \left\langle V_{2}, V_{3}\right\rangle & \left\langle V_{3}, V_{3}\right\rangle
\end{array}\right]
$$

Esta propriedade pode ser facilmente demonstrada lembrando que se $A=\left(a_{i j}\right)$ então $A^{*}=\left(\bar{a}_{j i}\right), \mathrm{e}$, que as colunas de $A$ são formadas pelos vetores $V_{1}, V_{2}$ e $V_{3}$.

\section{Produto vetorial hermitiano}

No espaço vetorial complexo $\mathbb{C}^{2,1}$, o produto vetorial hermitiano tem papel semelhante ao do produto vetorial usual no espaço euclidiano $\mathbb{R}^{3}$. Sandler ([2], p. 103) apresenta o produto vetorial hermitiano como $Z \otimes W=H(\bar{Z} \times \bar{W})$, em que $Z, W \in \mathbb{C}^{2,1}, \mathrm{H}$ é a matriz da forma hermitiana, $Z \times W$ é o produto vetorial ordinário e as barras denotam o conjugado complexo. Assim, para cada forma hermitiana obtemos uma expressão para o produto vetorial hermitiano.

Consideraremos a forma hermitiana usual para a definição e demonstração das propriedades do produto vetorial hermitiano. Considere $\mathbb{C}^{2,1}$ munido da forma hermitiana $\langle Z, W\rangle=z_{1} \overline{w_{1}}+z_{2} \overline{w_{2}}-z_{3} \overline{w_{3}}$ para $Z=\left[\begin{array}{c}z_{1} \\ z_{2} \\ z_{3}\end{array}\right]$ e $W=\left[\begin{array}{c}w_{1} \\ w_{2} \\ w_{3}\end{array}\right] \in \mathbb{C}^{2,1}$.

Definição 2.1. O produto vetorial hermitiano é dado pela função

$$
\otimes: \mathbb{C}^{2,1} \times \mathbb{C}^{2,1} \rightarrow \mathbb{C}^{2,1}
$$


definida por:

$$
Z \otimes W=\left[\begin{array}{l}
z_{1} \\
z_{2} \\
z_{3}
\end{array}\right] \otimes\left[\begin{array}{c}
w_{1} \\
w_{2} \\
w_{3}
\end{array}\right]=\left[\begin{array}{l}
\overline{z_{2}} \cdot \overline{w_{3}}-\overline{z_{3}} \cdot \overline{w_{2}} \\
\overline{z_{3}} \cdot \overline{w_{1}}-\overline{z_{1}} \cdot \overline{w_{3}} \\
\overline{z_{2}} \cdot \overline{w_{1}}-\overline{z_{1}} \cdot \overline{w_{2}}
\end{array}\right]
$$

Como no caso real em $\mathbb{R}^{3}$, o vetor $Z \otimes W$ é ortogonal a $Z$ e a $W$, isto é,

$$
\langle Z, Z \otimes W\rangle=\langle W, Z \otimes W\rangle=0
$$

Lema 2.1. Sejam $P, Q, R, S \in \mathbb{C}^{2,1}$. Então:

1. $P \otimes(Q \otimes R)=\langle Q, P\rangle R-\langle R, P\rangle Q$

2. $\langle P \otimes Q, R \otimes S\rangle=\langle S, P\rangle\langle R, Q\rangle-\langle R, P\rangle\langle S, Q\rangle$

3. Se $P$ e $Q$ são vetores nulos, isto é, pertencentes a $V_{0}, \mathrm{e}, P, Q \in \mathbb{C}^{2,1}-\{0\}$, com $P \neq Q$, então $P \otimes Q$ é um vetor positivo ortogonal a $P$ e a $Q$ tal que $\langle P \otimes Q, P \otimes Q\rangle=|\langle P, Q\rangle|^{2}$

Demonstração. Usaremos a seguinte forma hermitiana $\langle P, Q\rangle=p_{1} \overline{q_{1}}+p_{2} \overline{q_{2}}-p_{3} \overline{q_{3}}$ $\operatorname{com} P=\left[\begin{array}{l}p_{1} \\ p_{2} \\ p_{3}\end{array}\right], Q=\left[\begin{array}{l}q_{1} \\ q_{2} \\ q_{3}\end{array}\right], R=\left[\begin{array}{l}r_{1} \\ r_{2} \\ r_{3}\end{array}\right]$ e $S=\left[\begin{array}{l}s_{1} \\ s_{2} \\ s_{3}\end{array}\right]$.

1. Pela definição obtemos:

$$
\begin{aligned}
P \otimes(Q \otimes R) & =\left[\begin{array}{l}
p_{1} \\
p_{2} \\
p_{3}
\end{array}\right] \otimes\left[\begin{array}{l}
\overline{q_{2}} \cdot \overline{r_{3}}-\overline{q_{3}} \cdot \overline{r_{2}} \\
\overline{q_{3}} \cdot \overline{r_{1}}-\overline{q_{1}} \cdot \overline{r_{3}} \\
\overline{q_{2}} \cdot \overline{r_{1}}-\overline{q_{1}} \cdot \overline{r_{2}}
\end{array}\right] \\
& =\left[\begin{array}{l}
\overline{p_{2}}\left(q_{2} r_{1}-q_{1} r_{2}\right)-\overline{p_{3}}\left(q_{1} r_{3}-q_{3} r_{1}\right) \\
\overline{p_{3}}\left(q_{2} r_{3}-q_{3} r_{2}\right)-\overline{p_{1}}\left(q_{1} r_{2}-q_{2} r_{1}\right) \\
\overline{p_{2}}\left(q_{2} r_{3}-q_{3} r_{2}\right)-\overline{p_{1}}\left(q_{1} r_{3}-q_{3} r_{1}\right)
\end{array}\right]
\end{aligned}
$$

Calculando $\langle Q, P\rangle R-\langle R, P\rangle Q$ temos:

$$
\langle Q, P\rangle R-\langle R, P\rangle Q=\left[\begin{array}{l}
\overline{p_{2}}\left(q_{2} r_{1}-q_{1} r_{2}\right)-\overline{p_{3}}\left(q_{1} r_{3}-q_{3} r_{1}\right) \\
\overline{p_{3}}\left(q_{2} r_{3}-q_{3} r_{2}\right)-\overline{p_{1}}\left(q_{1} r_{2}-q_{2} r_{1}\right) \\
\overline{p_{2}}\left(q_{2} r_{3}-q_{3} r_{2}\right)-\overline{p_{1}}\left(q_{1} r_{3}-q_{3} r_{1}\right)
\end{array}\right]
$$

Por (2.4) e (2.5) vemos que $P \otimes(Q \otimes R)=\langle Q, P\rangle R-\langle R, P\rangle Q$. 
2. Por definição, $P \otimes Q=\left[\begin{array}{l}\overline{p_{2}} \cdot \overline{q_{3}}-\overline{p_{3}} \cdot \overline{q_{2}} \\ \overline{p_{3}} \cdot \overline{q_{1}}-\overline{p_{1}} \cdot \overline{q_{3}} \\ \overline{p_{2}} \cdot \overline{q_{1}}-\overline{p_{1}} \cdot \overline{q_{2}}\end{array}\right]$ e $R \otimes S=\left[\begin{array}{l}\overline{r_{2}} \cdot \overline{s_{3}}-\overline{r_{3}} \cdot \overline{s_{2}} \\ \overline{r_{3}} \cdot \overline{s_{1}}-\overline{r_{1}} \cdot \overline{s_{3}} \\ \overline{r_{2}} \cdot \overline{s_{1}}-\overline{r_{1}} \cdot \overline{s_{2}}\end{array}\right]$, então: $\langle P \otimes Q, R \otimes S\rangle=$

$$
\begin{aligned}
& \overline{p_{2}} \cdot \overline{q_{3}} r_{2} s_{3}-\overline{p_{2}} \cdot \overline{q_{3}} r_{3} s_{2}-\overline{p_{3}} \cdot \overline{q_{2}} r_{2} s_{3}+\overline{p_{3}} \cdot \overline{q_{2}} r_{3} s_{2} \\
& +\overline{p_{3}} \cdot \overline{q_{1}} r_{3} s_{1}-\overline{p_{3}} \cdot \overline{q_{1}} r_{1} s_{3}-\overline{p_{1}} \cdot \overline{q_{3}} r_{3} s_{1}+\overline{p_{1}} \cdot \overline{q_{3}} r_{1} s_{3} \\
& -\overline{p_{2}} \cdot \overline{q_{1}} r_{2} s_{1}+\overline{p_{2}} \cdot \overline{q_{1}} r_{1} s_{3}+\overline{p_{1}} \cdot \overline{q_{2}} r_{2} s_{1}-\overline{p_{1}} \cdot \overline{q_{2}} r_{1} s_{3}
\end{aligned}
$$

Calculando $\langle S, P\rangle\langle R, Q\rangle-\langle R, P\rangle\langle S, Q\rangle$ obtemos:

$$
\begin{aligned}
& \langle S, P\rangle\langle R, Q\rangle-\langle R, P\rangle\langle S, Q\rangle= \\
& \quad\left(s_{1} \overline{p_{1}}+s_{2} \overline{p_{2}}-s_{3} \overline{p_{3}}\right)\left(r_{1} \overline{q_{1}}+r_{2} \overline{q_{2}}-r_{3} \overline{q_{3}}\right) \\
& -\left(r_{1} \overline{p_{1}}+r_{2} \overline{p_{2}}-r_{3} \overline{p_{3}}\right)\left(s_{1} \overline{q_{1}}+s_{2} \overline{q_{2}}-s_{3} \overline{q_{3}}\right)
\end{aligned}
$$

Resolvendo (2.7) encontramos (2.6). Segue que $\langle P \otimes Q, R \otimes S\rangle=\langle S, P\rangle\langle R, Q\rangle-$ $\langle R, P\rangle\langle S, Q\rangle$.

3. Para provar que $\langle P, Q\rangle \neq 0$ usaremos a seguinte propriedade: se $P, Q \in$ $\mathbb{C}^{2,1}-\{0\}, \operatorname{com}\langle P, P\rangle \leq 0$ e $\langle Q, Q\rangle \leq 0$, então $P=\lambda Q$, para algum $\lambda \in \mathbb{C}^{2,1}$, ou $\langle P, Q\rangle \neq 0$.

Se $P$ e $Q$ são vetores nulos, e, $P, Q \in \mathbb{C}^{2,1}-\{0\}, \operatorname{com} P \neq Q$, então $\langle P, P\rangle=0=$ $\langle Q, Q\rangle$, e, pela propriedade enunciada, $\langle P, Q\rangle \neq 0$. Além disso, $|\langle P, Q\rangle|^{2}=$ $\langle P, Q\rangle\langle Q, P\rangle$. Assim, do item anterior segue que:

$$
\langle P \otimes Q, P \otimes Q\rangle=\langle Q, P\rangle\langle P, Q\rangle-\langle P, P\rangle\langle Q, Q\rangle=|\langle P, Q\rangle|^{2}
$$

Observação 2.1. Considere $Z=\left[\begin{array}{c}z_{1} \\ z_{2} \\ z_{3}\end{array}\right]$ e $W=\left[\begin{array}{c}w_{1} \\ w_{2} \\ w_{3}\end{array}\right] \in \mathbb{C}^{2,1}$.

- Se em $\mathbb{C}^{2,1}$ é considerada a forma hermitiana $\langle Z, W\rangle=z_{1} \overline{w_{3}}+z_{2} \overline{w_{2}}+z_{3} \overline{w_{1}}$ dada pela matriz $\mathrm{H}_{2}$, então o produto vetorial hermitiano é definido por

$$
\mathrm{Z} \otimes=\left[\begin{array}{c}
z_{1} \\
z_{2} \\
z_{3}
\end{array}\right] \otimes\left[\begin{array}{c}
w_{1} \\
w_{2} \\
w_{3}
\end{array}\right]=\left[\begin{array}{l}
\overline{z_{1}} \cdot \overline{w_{2}}-\overline{z_{2}} \cdot \overline{w_{1}} \\
\overline{z_{3}} \cdot \overline{w_{1}}-\overline{z_{1}} \cdot \overline{w_{3}} \\
\overline{z_{2}} \cdot \overline{w_{3}}-\overline{z_{3}} \cdot \overline{w_{2}}
\end{array}\right]
$$


- Se em $\mathbb{C}^{2,1}$ é considerada a forma hermitiana $\langle Z, W\rangle=z_{1} \overline{w_{1}}-z_{2} \overline{w_{3}}-z_{3} \overline{w_{2}}$ dada pela matriz $\mathrm{H}_{3}$, o produto vetorial hermitiano é definido por

$$
\mathrm{Z} \otimes=\left[\begin{array}{l}
z_{1} \\
z_{2} \\
z_{3}
\end{array}\right] \otimes\left[\begin{array}{l}
w_{1} \\
w_{2} \\
w_{3}
\end{array}\right]=\left[\begin{array}{l}
\overline{z_{2}} \cdot \overline{w_{3}}-\overline{z_{3}} \cdot \overline{w_{2}} \\
\overline{z_{2}} \cdot \overline{w_{1}}-\overline{z_{1}} \cdot \overline{w_{2}} \\
\overline{z_{1}} \cdot \overline{w_{3}}-\overline{z_{3}} \cdot \overline{w_{1}}
\end{array}\right]
$$

- Independente da forma hermitiana $H_{1}, H_{2}$ ou $H_{3}$ definida em $\mathbb{C}^{2,1}$, são válidas as propriedades enunciadas para o produto vetorial hermitiano. Para mais detalhes ver ([3], p. 11).

Vamos utilizar o conceito e as propriedades do produto vetorial hermitiano para demonstrar que o grupo $P U(2,1)$ age duplamente transitivamente em $\partial \mathbb{H}_{\mathbb{C}}^{2}$. Mas antes é necessário a apresentação do seguinte lema:

Lema 2.2. Seja $V$ o conjunto dos pares $(P, Q)$ de vetores nulos em $\mathbb{C}^{2,1}$ tais que $\langle P, Q\rangle=-1$. O conjunto $U(2,1)$ age transitivamente no conjunto $V$.

Demonstração. Em $\mathbb{C}^{2,1}$ vamos considerar a forma hermitiana dada pela matriz $\mathrm{H}_{3}$, ou seja, $\langle Z, W\rangle=z_{1} \overline{w_{1}}-z_{2} \overline{w_{3}}-z_{3} \overline{w_{2}}, Z, W \in \mathbb{C}^{2,1}$.

Observe que se $A=\left[\begin{array}{l}0 \\ 1 \\ 0\end{array}\right]$ e $B=\left[\begin{array}{l}0 \\ 0 \\ 1\end{array}\right]$, então $A$ e $B$ são vetores nulos em $\mathbb{C}^{2,1}$ tais que $\langle A, B\rangle=-1$. Logo, o par $(A, B)$ pertence ao conjunto $V$.

Sejam agora $P$ e $Q$ vetores nulos em $\mathbb{C}^{2,1}$ tais que $\langle P, Q\rangle=-1$. Ou seja, $(P, Q)$ pertence ao conjunto $V$. Para demonstrar esse lema vamos exibir uma matriz $M \in U(2,1)$ tal que $M \cdot A=P$ e $M \cdot B=Q$.

De fato, considere a matriz $M_{3 \times 3}$ cujas colunas são, respectivamente, os vetores $P \otimes Q, P$ e $Q$. Pela propriedade 1.1 temos que:

$$
\begin{aligned}
M^{*} H_{3} M & =\left[\begin{array}{ccc}
\langle P \otimes Q, P \otimes Q\rangle & \langle P, P \otimes Q\rangle & \langle Q, P \otimes Q\rangle \\
\langle P \otimes Q, P\rangle & \langle P, P\rangle & \langle Q, P\rangle \\
\langle P \otimes Q, Q\rangle & \langle P, Q\rangle & \langle Q, Q\rangle
\end{array}\right] \\
M^{*} H_{3} M & =\left[\begin{array}{ccc}
\left.\langle P, Q\rangle\right|^{2} & 0 & 0 \\
0 & 0 & -1 \\
0 & -1 & 0
\end{array}\right]=\left[\begin{array}{ccc}
1 & 0 & 0 \\
0 & 0 & -1 \\
0 & -1 & 0
\end{array}\right]=H_{3}
\end{aligned}
$$

Assim, $M^{*} H_{3} M=H_{3}$ e, portanto, $M \in U(2,1)$.

Como $M \cdot A=P$ e $M \cdot B=Q$ segue que o conjunto $U(2,1)$ age transitivamente no conjunto $V$. 
Proposição 2.1. O grupo $P U(2,1)$ age duplamente transitivamente em $\partial \mathbb{H}_{\mathbb{C}}^{2}$.

Demonstração. Sejam $p_{1}$ e $q_{1}$ dois pontos distintos em $\partial \mathbb{H}_{\mathbb{C}}^{2}$ e $p_{2}$ e $q_{2}$ dois pontos distintos em $\partial \mathbb{H}_{\mathbb{C}}^{2}$.

Considere $P_{1}, Q_{1}, P_{2}, Q_{2}$ levantamentos de $p_{1}, q_{1}, p_{2}$ e $q_{2}$, respectivamente, normalizados de modo que $\left\langle P_{1}, Q_{1}\right\rangle=-1$ e $\left\langle P_{2}, Q_{2}\right\rangle=-1$.

O lema anterior garante que existe uma matriz $M \in U(2,1)$ tal que $M \cdot P_{1}=P_{2}$ e $M \cdot Q_{1}=Q_{2}$. Fazendo a projeção obtemos $f \in P U(2,1)$ tal que $f\left(p_{1}\right)=p_{2}$ e $f\left(q_{1}\right)=q_{2}$, isto é, o grupo $P U(2,1)$ age duplamente transitivamente em $\partial \mathbb{H}_{\mathbb{C}}^{2}$.

É possível demonstrar a proposição anterior usando a forma hermitiana $\mathrm{H}_{2}$ como apresentado por Parker ([3], p. 13), e mostrar que PU(2,1) age transitivamente no espaço hiperbólico complexo de dimensão 2 (para essa demonstração veja [3], p. 12).

Como mostra ([5], p. 10), pode-se encontrar uma matriz $N_{3 \times 3}, \operatorname{com~} N^{*} H_{1} N=H_{1}$, de modo que $N=D M D^{-1}$, em que $M$ é a matriz apresentada no lema 2.2, e $D$ é a matriz de mudança de base, da base de $\mathbb{C}^{3}$ que gera a forma hermitiana $\mathrm{H}_{3}$ para a base de $\mathbb{C}^{3}$ que gera a forma hermitiana $H_{1}$. Ou seja, o lema 2.2 pode ser demostrado usando a forma hermitiana $H_{1}$.

\section{Geodésicas}

No espaço hiperbólico complexo podemos definir dois tipos de subvariedades totalmente geodésicas, as subvariedades totalmente geodésicas complexas e as subvariedades totalmente geodésicas e totalmente reais. As subvariedades totalmente geodésicas complexas de $\mathbb{H}_{\mathbb{C}}^{2}$ são equivalentes, por isometrias, a $\mathbb{H}_{\mathbb{C}}^{k}$ onde $k \in\{1,2\}$. Nessa classe de subvariedades definiremos as geodésicas complexas. Já as subvariedades totalmente geodésicas e totalmente reais de $\mathbb{H}_{\mathbb{C}}^{2}$ são equivalentes, por isometrias, a $\mathbb{H}_{\mathbb{R}}^{k}$ sendo $k \in\{1,2\}$. Neste último caso, quando $k=2$, chamamos a subvariedade totalmente geodésica e totalmente real de $\mathbb{R}^{2}$-plano e a interseção de um $\mathbb{R}^{2}$-plano com $\partial \mathbb{H}_{\mathbb{C}}^{2}$ chamamos de um $\mathbb{R}$-círculo. Aqui apresentaremos apenas as definições de geodésica real e geodésica complexa. 


\subsection{Linha geodésica real}

Sejam $p$ e $q$ dois pontos distintos em $\partial \mathbb{H}_{\mathbb{C}}^{2}$. Vamos determinar uma parametrização para a linha geodésica real (ou geodésica) em $\mathbb{H}_{\mathbb{C}}^{2}$ com pontos finais $p$ e $q$. Para isso vamos considerar o modelo da bola $\mathbb{B}^{2}$ para o espaço hiperbólico complexo. Escolha levantamentos $P$ e $Q$ de $p$ e $q$, respectivamente, para vetores nulos em $\mathbb{C}^{2,1}$ normalizados de modo que $\langle P, Q\rangle=-1$.

Considere agora $a=\left[\begin{array}{l}1 \\ 0\end{array}\right]$ e $b=\left[\begin{array}{c}-1 \\ 0\end{array}\right]$ pontos em $\partial \mathbb{H}_{\mathbb{C}}^{2}$. A linha geodésica ligando esses pontos é dada por $\lambda(t)=\left[\begin{array}{l}t \\ 0\end{array}\right],-1<t<1$, ou equivalentemente, por $\lambda(t)=\left[\begin{array}{c}\tanh (t) \\ 0\end{array}\right], t \in \mathbb{R}$.

Em $\mathbb{C}^{2,1}$ considere levantamentos $A=\left[\begin{array}{c}\sqrt{2} / 2 \\ 0 \\ \sqrt{2} / 2\end{array}\right]$ e $B=\left[\begin{array}{c}-\sqrt{2} / 2 \\ 0 \\ \sqrt{2} / 2\end{array}\right]$ de $a$ e $b$ para vetores nulos tais que $\langle A, B\rangle=-1$. Levantando cada um dos pontos $\lambda(t)$, vemos que esta geodésica é representada pelo caminho de vetores negativos em $\mathbb{C}^{2,1}$ :

$$
\begin{aligned}
\tilde{\lambda}(t) & =\left[\begin{array}{c}
\sinh (t) \\
0 \\
\cosh (t)
\end{array}\right]=\left[\begin{array}{c}
\frac{e^{t}-e^{-t}}{2} \\
0 \\
\frac{e^{t}+e^{-t}}{2}
\end{array}\right]= \\
& =\frac{e^{t}}{\sqrt{2}}\left[\begin{array}{c}
\sqrt{2} / 2 \\
0 \\
\sqrt{2} / 2
\end{array}\right]+\frac{e^{-t}}{\sqrt{2}}\left[\begin{array}{c}
-\sqrt{2} / 2 \\
0 \\
\sqrt{2} / 2
\end{array}\right]= \\
& =\frac{e^{t}}{\sqrt{2}} A+\frac{e^{-t}}{\sqrt{2}} B=\frac{1}{\sqrt{2}}\left(e^{t} A+e^{-t} B\right)
\end{aligned}
$$

Como $A$ e $B$ são vetores nulos tais que $\langle A, B\rangle=-1$, e, como $P$ e $Q$ também são vetores nulos tais que $\langle P, Q\rangle=-1$, pelo lema 2.2 existe uma matriz $M \in U(2,1)$ tal que $M \cdot A=P$ e $M \cdot B=Q$.

Já que $M$ induz uma isometria $f \in P U(2,1)$ tal que $f(a)=p$ e $f(b)=q$, e como isometria transforma linhas geodésicas em linhas geodésicas, temos que $f(\lambda(t))$ é a geodésica com pontos finais $p$ e $q$.

No levantamento esta geodésica é representada por

$$
M \cdot \widetilde{\lambda}(t)=\frac{1}{\sqrt{2}}\left(e^{t} M \cdot A+e^{-t} M \cdot B\right)=\frac{1}{\sqrt{2}}\left(e^{t} P+e^{-t} Q\right)
$$


O que fizemos demonstra a seguinte proposição:

Proposição 3.1. Sejam $p$ e $q$ pontos distintos em $\partial \mathbb{H}_{\mathbb{C}}^{2}$ representados por vetores nulos $P$ e $Q$ em $\mathbb{C}^{2,1}$ tais que $\langle P, Q\rangle=-1$. A linha geodésica em $\mathbb{H}_{\mathbb{C}}^{2}$ com pontos finais $p$ e $q$ é parametrizada pelos pontos $a_{t}, t \in \mathbb{R}$, cujos levantamentos em $\mathbb{C}^{2,1}$ são dados por:

$$
A_{t}=\frac{1}{\sqrt{2}}\left(e^{t} P+e^{-t} Q\right)
$$

Observação 3.1. Qualquer par $z, w \in \partial \mathbb{H}_{\mathbb{C}}^{2}$ pertence a uma única geodésica.

Como mostra Parker ([3], p. 30), a parametrização que vimos para uma geodésica em $\mathbb{H}_{\mathbb{C}}^{2}$ é uma parametrização pelo comprimento de arco $t \in \mathbb{R}$, ou seja, usando a métrica de Bergman pode-se mostrar que a distância entre dois pontos na geodésica corresponde a uma distância real.

\subsection{Geodésicas Complexas}

Uma geodésica complexa é uma subvariedade totalmente geodésica complexa de dimensão complexa 1.

Definição 3.1. Seja $W \subset \mathbb{C}^{2,1}$ um subespaço complexo bidimensional. Suponha que $W \cap V_{-} \neq \varnothing$. Ao subespaço $\Sigma=\mathbb{P}\left(W \cap V_{-}\right)$de $\mathbb{H}_{\mathbb{C}}^{2}$ chamamos geodésica complexa.

Para quaisquer $z, w \in \mathbb{H}_{\mathbb{C}}^{2} \cup \partial \mathbb{H}_{\mathbb{C}}^{2}$ distintos, existe uma única geodésica complexa contendo $z$ e $w$. Dizemos que esses dois pontos geram a geodésica complexa. Para uma demonstração desse fato veja ([1], p. 75).

A interseção de uma geodésica complexa com $\partial \mathbb{H}_{\mathbb{C}}^{2}$ é chamada de cadeia.

Seja $P$ um vetor positivo em $\mathbb{C}^{2,1}$. Se $W=\left\{Z \in \mathbb{C}^{2,1} \mid\langle Z, P\rangle=0\right\}$, dizemos que $P$ é vetor polar.

Proposição 3.2. Toda geodésica complexa em $\mathbb{H}_{\mathbb{C}}^{2}$ pode ser dada por um vetor polar.

Demonstração. Seja $W \in \mathbb{C}^{2,1}$ um subespaço complexo bidimensional tal que $W \cap$ $V_{-} \neq \varnothing$. Sabe-se que o complemento ortogonal $W^{\perp}$ de $W$ em $\mathbb{C}^{2,1}$ é um subespaço de dimensão complexa 1 de modo que todo vetor não nulo de $W^{\perp}$ é um vetor positivo. Assim, qualquer vetor positivo em $W^{\perp}$ é um vetor polar da geodésica complexa $\Sigma=\mathbb{P}\left(W \cap V_{-}\right)$, isto é, dado $P$ um vetor positivo em $W^{\perp}$ temos $\langle P, Q\rangle=0$, para qualquer $Q \in W$.

Reciprocamente, dado qualquer vetor positivo $P \in \mathbb{C}^{2,1}$, se $W=\left\{Z \in \mathbb{C}^{2,1} \mid\langle Z, P\rangle=\right.$ $0\}$, então $W$ é um subespaço complexo bidimensional tal que $W \cap V_{-} \neq \varnothing$ e $\Sigma=\mathbb{P}\left(W \cap V_{-}\right)$é uma geodésica complexa de vetor polar $P$. 
Como veremos logo a seguir, podemos utilizar vetores polares à geodésicas complexas para determinar suas posições relativas.

\section{Classificação de pares de geodésicas complexas:}

Sejam $\Sigma_{1}$ e $\Sigma_{2}$ duas geodésicas complexas distintas que correspondem a vetores polares $P, Q \in \mathbb{C}^{2,1}$, respectivamente. Considere o produto vetorial hermitiano $W=P \otimes Q$. De acordo com Goldman ([1], p. 100), existem três possibilidades:

1. W é positivo:

As geodésicas complexas $\Sigma_{1}$ e $\Sigma_{2}$ são ultraparalelas, isto é, são disjuntas e tem uma única geodésica complexa $\Sigma$ ortogonal comum com vetor polar $W$.

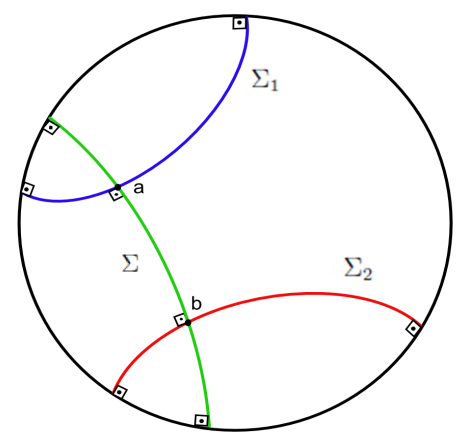

Figura 1: Geodésicas complexas ultraparalelas

Se $a=\Sigma \cap \Sigma_{1}$ e $b=\Sigma \cap \Sigma_{2}$ então esses pontos possuem levantamentos $A=P \otimes(P \otimes Q)$ e $B=Q \otimes(P \otimes Q)$ e, por definição, $\rho\left(\Sigma_{1}, \Sigma_{2}\right)=\rho(a, b)$. Assim,

$$
\cosh ^{2}\left(\frac{\rho\left(\Sigma_{1}, \Sigma_{2}\right)}{2}\right)=\frac{\langle A, B\rangle\langle B, A\rangle}{\langle A, A\rangle\langle B, B\rangle}
$$

Utilizando o lema 2.1 pode ser verificado que a distância entre as geodésicas complexas $\Sigma_{1}$ e $\Sigma_{2}$ também é expressa em termos dos vetores polares $P$ e $Q$ como:

$$
\cosh ^{2}\left(\frac{\rho\left(\Sigma_{1}, \Sigma_{2}\right)}{2}\right)=\frac{\langle P, Q\rangle\langle Q, P\rangle}{\langle P, P\rangle\langle Q, Q\rangle}
$$

2. W é nulo:

As geodésicas complexas $\Sigma_{1}$ e $\Sigma_{2}$ são assintóticas ou paralelas, ou seja, se intersectam no ponto $\mathbb{P}(W) \in \partial \mathbb{H}_{\mathbb{C}}^{2}$. 


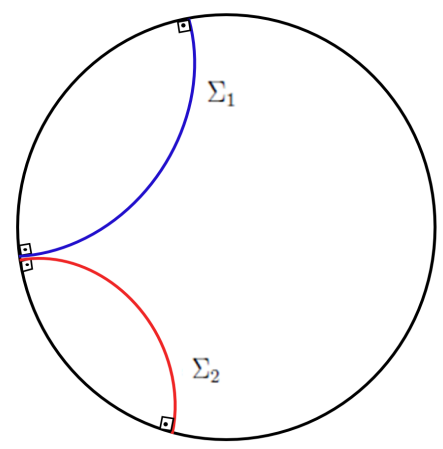

Figura 2: Geodésicas complexas paralelas

3. W é negativo:

As geodésicas complexas $\Sigma_{1}$ e $\Sigma_{2}$ são concorrentes, isto é, elas se intersectam no ponto $\mathbb{P}(W) \in \mathbb{H}_{\mathbb{C}}^{2}$.

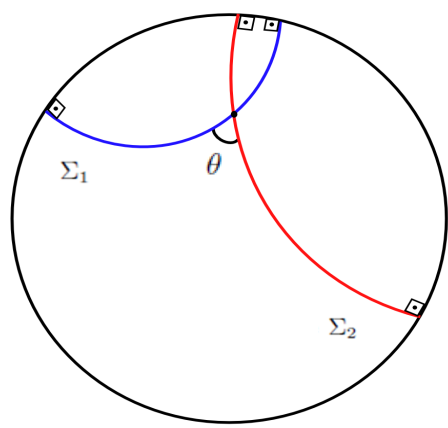

Figura 3: Geodésicas complexas concorrentes

Podemos definir o ângulo $\theta \in[0, \pi / 2]$ entre as geodésicas complexas $\Sigma_{1}$ e $\Sigma_{2}$ em termos de vetores polares como segue:

$$
\cos ^{2}(\theta)=\frac{\langle P, Q\rangle\langle Q, P\rangle}{\langle P, P\rangle\langle Q, Q\rangle}
$$

Resumindo, se $\Sigma_{1}$ e $\Sigma_{2}$ são geodésicas complexas em $\mathbb{H}_{\mathbb{C}}^{2}$ com vetores polares $P, Q \in \mathbb{C}^{2,1}$, respectivamente, então:

- $\Sigma_{1}$ e $\Sigma_{2}$ são ultraparalelas $\Leftrightarrow \frac{\langle P, Q\rangle\langle Q, P\rangle}{\langle P, P\rangle\langle Q, Q\rangle}>1$.

- $\Sigma_{1}$ e $\Sigma_{2}$ são assintóticas $\Leftrightarrow \frac{\langle P, Q\rangle\langle Q, P\rangle}{\langle P, P\rangle\langle Q, Q\rangle}=1$. 
- $\Sigma_{1}$ e $\Sigma_{2}$ são concorrentes $\Leftrightarrow \frac{\langle P, Q\rangle\langle Q, P\rangle}{\langle P, P\rangle\langle Q, Q\rangle}<1$.

\section{Considerações finais}

Conhecer os objetos do espaço hiperbólico complexo permite encontrar características importantes desse espaço como, por exemplo, as distâncias entre esses objetos. Um outro elemento importante do espaço hiperbólico complexo são os bissetores que, apesar de não serem subvariedades totalmente geodésicas em $\mathbb{H}_{\mathbb{C}^{\prime}}^{2}$ podem ser decompostos em subvariedades totalmente geodésicas.

É possível, apesar de na maioria das vezes não ser fácil, encontrar fórmulas de distâncias entre esses objetos de $\mathbb{H}_{\mathbb{C}}^{2}$. Goldman ([1], p. 76) apresenta a métrica de Bergman como uma fórmula de distância entre dois pontos $p, q \in \mathbb{H}_{\mathbb{C}}^{2}$. Utilizando a projeção ortogonal sobre uma geodésica complexa conseguimos, através da métrica de Bergman, encontrar uma fórmula para a distância entre um ponto em $\mathbb{H}_{\mathbb{C}}^{2}$ e uma geodésica complexa. Baseado nestas duas fórmulas e considerando a definição do $\eta$-invariante, que é um invariante no espaço hiperbólico complexo, encontramos fórmulas de distância para combinações entre pontos e qualquer outro desses objetos em $\mathbb{H}_{\mathbb{C}^{\prime}}^{2}$ e entre geodésicas complexas e qualquer outro desses elementos em $\mathbb{H}_{\mathbb{C}}^{2}$. No entanto, para distância entre duas linhas geodésicas reais, entre dois bissetores e entre uma geodésica e um bissetor, o problema fica mais complexo, pois envolve a extração de raízes de polinômios de grau 6 cujos coeficientes estão em função da forma hermitiana, e tais polinômios podem não ser solúveis por radicais em uma extensão transcendental $F$ de $\mathbb{Q}$. Assim, dependendo do polinômio envolvido, nem sempre será possível explicitar a distância entre geodésicas e bissetores. As fórmulas de distâncias no espaço hiperbólico complexo podem ser encontradas em [6].

\section{Referências}

[1] GOLDMAN, Willian M. Complex Hyperbolic Geometry. Oxford University Press, 1999.

[2] SANDLER, Hanna. Distance formulas in complex hyperbolic space. Forum Math. 8 (1996), nº 1, $93-106$.

[3] PARKER, John R. Notes on Complex Hyperbolic Geometry. 2010. 
[4] CONWAY, John B. Functions of one complex variable, $2^{\mathrm{a}}$ edição. New York: Springer-Verlag, 1978.

[5] LOPES, Aldo P. C. Um estudo da geometria hiperbólica complexa, 2009. Dissertação (Mestrado em Matemática) - Instituto de Ciências Exatas, Universidade Federal de Minas Gerais, Belo Horizonte.

[6] SANTANA, Joyce D. Fórmulas de distância no espaço hiperbólico complexo, 2014. Dissertação (Mestrado em Matemática) - Instituto de Ciências Exatas, Universidade Federal de Minas Gerais, Belo Horizonte.

[7] ABRANTES, Lia F. F. Configurações de pontos na fronteira e no interior do espaço hiperbólico real $\mathbb{H}_{\mathbb{R}^{\prime}}^{n}$ 2010. Dissertação (Mestrado em Matemática) - Instituto de Ciências Exatas, Universidade Federal de Minas Gerais, Belo Horizonte.

[8] JUNIOR, João dos R. Esferas hiperbólicas com pontos cônicos, 2010. Dissertação (Mestrado em Ciências) - Instituto de Ciências Matemáticas e Computação, Universidade de São Paulo, São Carlos. 